\title{
INTEGRAÇÃO DE DADOS DE GRAVIMETRIA MARINHA E DE ALTIMETRIA POR SATÉLITE (GEOSAT/ERM) NO ATLÂNTICO SUL $\left(65 / 25^{\circ} \mathrm{W}\right.$ E $\left.25 / 40^{\circ} \mathrm{S}\right)$.
}

\begin{abstract}
E. P. Leite, E. C. Molina \& N. Ussami
Foram integrados dados de gravimetria marinha convencional com aqueles derivados de altimetria por satélite na região do Atlântico Sul entre as coordenadas $65 / 25^{\circ} \mathrm{W}$ e 25 / $40^{\circ} \mathrm{S}$. Esta região inclui, entre outras feições fisiográficas e tectônicas, o sul do Platô de São Paulo, a Elevação do Rio Grande, a Bacia de Pelotas e o Cone do Rio Grande. Utilizando a colocação por mínimos quadrados, a anomalia ar-livre e a altura geoidal foram representadas na forma de modelos digitais com resolução nominal de 10'. Os dados de gravimetria convencional foram obtidos, em sua maioria, do banco de dados GEODAS atualizado até 1995 e os de altimetria por satélite são provenientes de um ano de rastreio da missão GEOSAT/Exact Repeat Mission. A representação das anomalias ar-livre feita neste trabalho foi comparada com duas outras representações recentes, que foram feitas baseadas somente em dados de altimetria por satélite de diferentes missões. Os resultados indicam que a metodologia empregada no presente estudo produz representações do campo de gravidade para a região oceânica isentas de ruídos de alta frequiência, uma característica comum em estimativas do campo que utilizam somente dados de altimetria por satélite. Além disso, a integração de dados de gravimetria convencional permite estimar melhor o campo gravitacional em regiões com lâmina de água menor do que $1.000 \mathrm{~m}$, e nas margens continentais, onde os dados de altimetria por satélite não são confiáveis. Como exemplo da potencialidade das representações das anomalias ar-livre e alturas geoidais obtidas, foi efetuada uma primeira análise qualitativa das principais anomalias com uma breve discussão do significado tectônico e implicações dinâmicas na evolução deste segmento sul da margem continental brasileira e litosfera oceânica adjacente.
\end{abstract}

Palavras-chave: Gravimetria Marinha; Atlântico Sul; Altimetria por Satélite.

INTEGRATION OF SHIPBORNE GRAVITY AND SATELLITE ALTIMETRY (GEOSAT/ERM) IN THE SOUTH ATLANTIC (65/25'W AND 25/40 ${ }^{\circ}$ S)-Conventional shipborne gravity data were integrated with gravity data derived from satellite altimetry over a region in the South Atlantic Ocean situated between $65 / 25^{\circ} \mathrm{W}$ and $25 / 40^{\circ} S$. This region includes physiographic and tectonic features such as part of the São Paulo Plateau, the Rio Grande Rise, the Pelotas Basin and the Rio Grande Fan. Least squares collocation was used in order to obtain free-air anomaly and geoid height models, with nominal resolution of 10'. Shipborne gravity data was mostly obtained from GEODAS data bank updated until 1995 and the satellite derived gravity data were measured during one year of GEOSAT/Exact Repeat Mission. The present free-air anomaly model was compared with two other recent models derived from different satellite altimetry missions. The methodology used in the present study produces noise-free gravity field models for the oceans in contrast with models based solely on satellite altimetry. Apart from this result, the integration of conventional marine gravity data is fundamental on improving the gravity field in regions of water depth shallower than 1,000 $\mathrm{m}$, such as over the continental margins, where satellite data are not reliable. As an example of the potentiality of the obtained free-air and geoid anomalies, a preliminary qualitative analysis of their tectonic significance is presented with emphasis on the dynamical implications on the evolution of the southern segment of the Brazilian continental margin and adjoining oceanic lithosphere.

Key words: Marine gravity; South Atlantic; Satellite Altimetry.

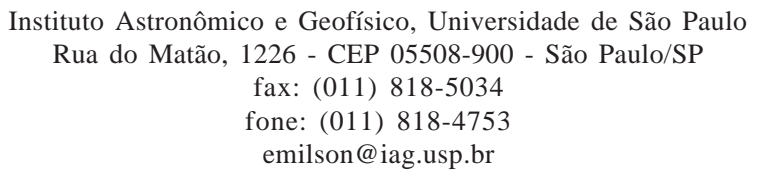




\section{INTRODUÇÃO}

A colocação por mínimos quadrados (Moritz, 1980) permite a utilização de dados heterogêneos na obtenção de estimativas de qualquer elemento associado ao campo de gravidade. Para tanto, as covariâncias entre todos os elementos envolvidos no cálculo devem ser conhecidas. Na prática, as covariâncias são obtidas a partir de uma função ajustada às covariâncias amostrais das anomalias ar-livre pois estas últimas observações são aquelas que apresentam maior quantidade e melhor distribuição geográfica. Esta metodologia já foi empregada na determinação do mapa gravimétrico do Brasil com resolução de $0,5^{\circ}$ (Sá et al., 1993), no modelo digital do geóide de alta resolução para o Estado de São Paulo (Sá, 1988) e na integração de dados de gravimetria marinha e de altimetria por satélite para todo o Oceano Atlântico Sul, com resolução nominal de 5' (Molina, 1996). Neste último trabalho foi demonstrada a conveniência de se integrar dados de gravimetria marinha convencional, principalmente no mapeamento gravimétrico em regiões próximas à costa e margem continental, onde os dados de altimetria por satélite têm uma grande incerteza devido à variação da superfície do mar ocasionada pela pequena lâmina d'água $(<1.000 \mathrm{~m})$ e problemas de órbita do satélite na transição continente-oceano (Cheney et al., 1987).

Neste trabalho são apresentados os resultados da utilização da colocação por mínimos quadrados na obtenção de uma nova representação do campo gravitacional para a região compreendida entre 65/ $25^{\circ} \mathrm{W}$ e $25 / 40^{\circ} \mathrm{S}$, no Atlântico Sul, com resolução nominal de 10'. Este reprocessamento deveu-se à incorporação de novos dados de gravimetria marinha com o objetivo de validar e melhor delinear algumas anomalias de curto comprimento de onda que haviam sido detectadas nesta região por Molina (1996). Em seguida esta representação é comparada com outras duas calculadas a partir de altimetria por satélite tão somente (Andersen \& Knudsen, 1995 e Sandwell \& Yale, 1994) e é feita uma breve discussão sobre a potencialidade dos dados obtidos em estudos litosféricos. A modelagem quantitativa da representação integrada com a finalidade de conhecer a distribuição de densidade e a estrutura térmica da litosfera nesta região é uma continuidade natural deste trabalho.

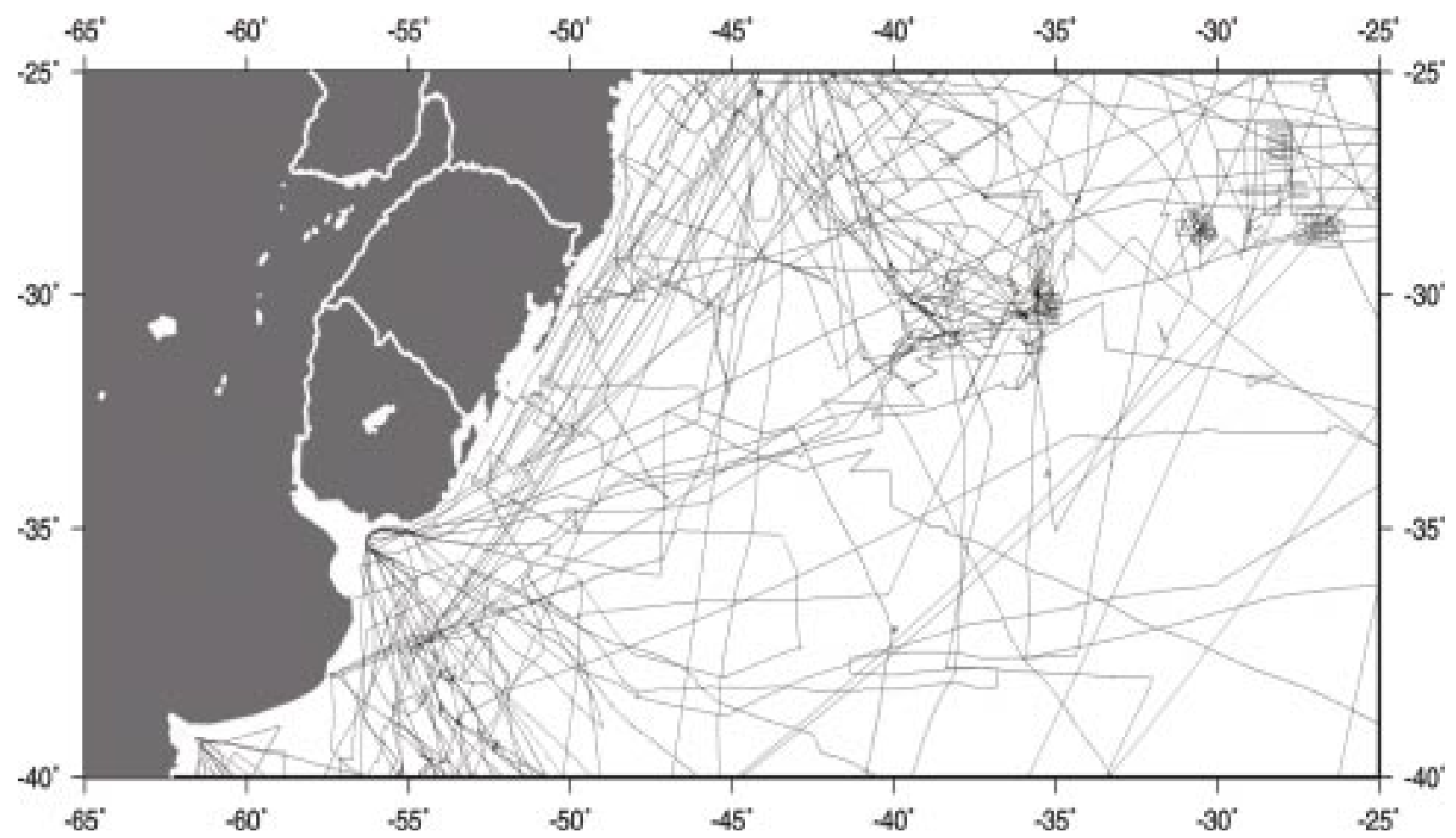

Figura 1 - Distribuição inicial de dados de gravimetria marinha e batimetria na região de estudo

Figure 1 - Marine gravity and bathymetry in the study region. 


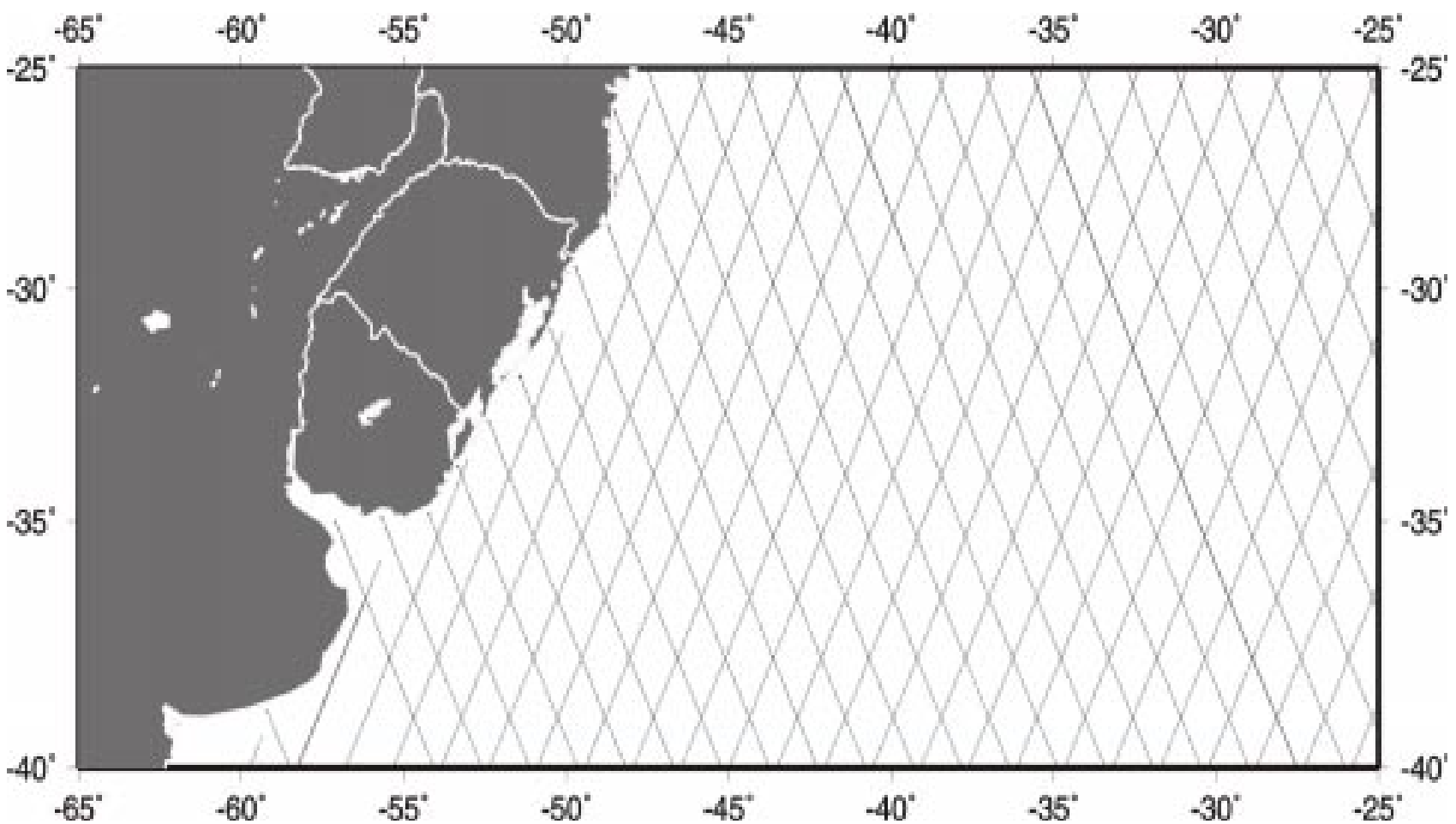

Figura 2 - Distribuição dos dados de altimetria por satélite da missão GEOSAT-ERM na região de estudo.

Figure 2 - GEOSAT/ERM Satellite altimetry tracks within the study region.

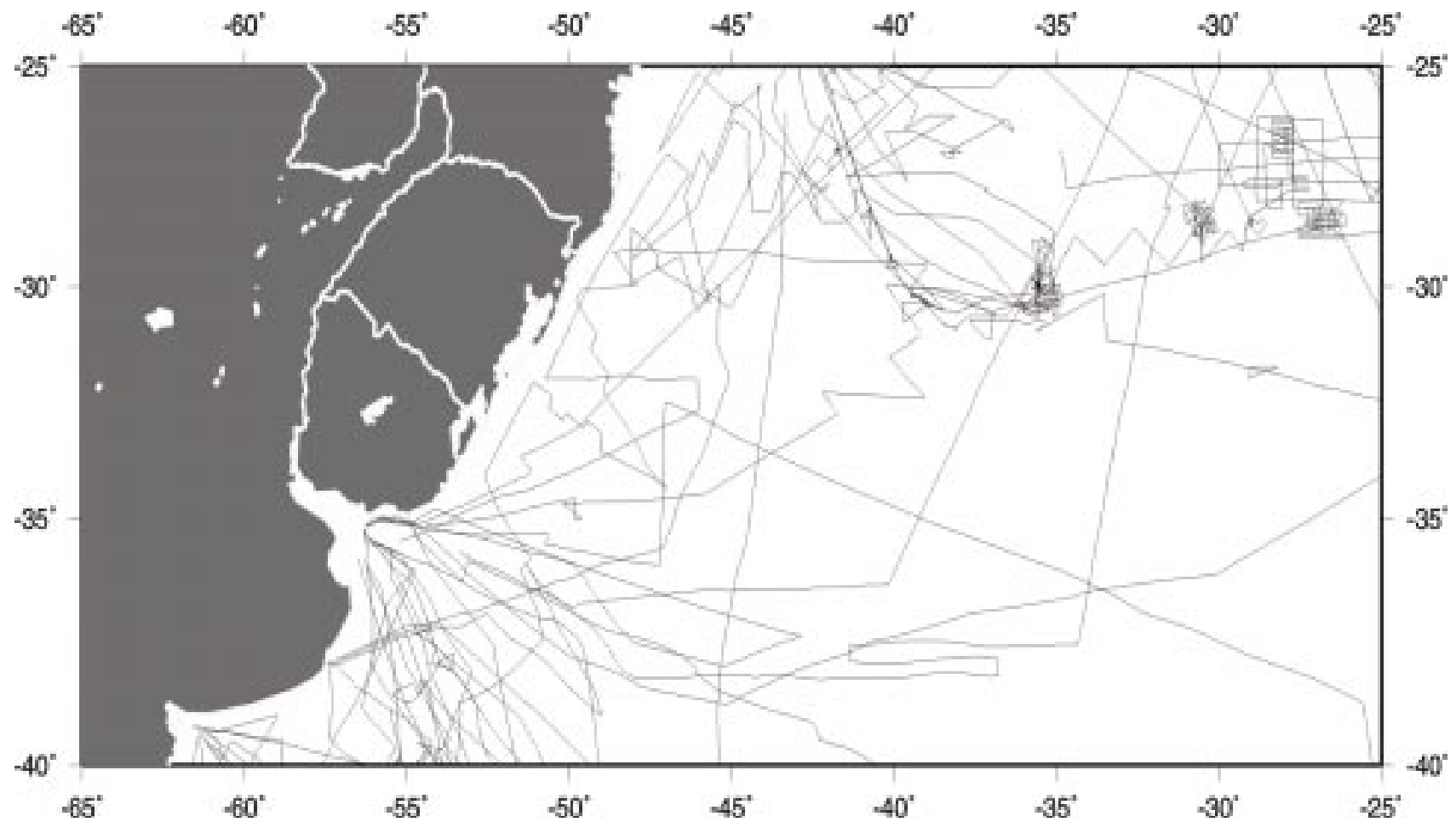

Figura 3 - Distribuição dos dados selecionados de gravimetria marinha e batimetria

Figure 3 - Selected gravity and bathymetric data. 


\section{DADOS UTILIZADOS}

\section{Geofísica marinha}

Os dados de geofísica marinha utilizados neste trabalho são provenientes do banco de dados do U. S. National Geophysical Data Center (NGDC, 1991). Para a região de estudo, estão disponíveis 388.273 pontos contendo dados de gravimetria, magnetometria, batimetria e sísmica. Os dados encontram-se no formato MGD77 (Hittleman et al., 1977).

Apesar da boa precisão dos dados de geofísica marinha, notam-se diferenças de cruzamento entre as rotas, que podem ser causadas por problemas instrumentais e metodológicos ou pelo processamento posterior dos dados. A análise dessas diferenças permite obter informações a respeito da precisão global do levantamento e possibilita o ajustamento entre os diversos levantamentos existentes em uma região. A Fig. 1 mostra a distribuição dos dados de geofísica marinha, gravimetria, magnetometria e batimetria, disponíveis na área de estudo.

\section{Altimetria por satélite}

As missões de altimetria por satélite fornecem dados com distribuição geográfica global e homogênea, auxiliando na determinação dos elementos do campo de gravidade terrestre principalmente em regiões onde não há outras fontes de informação. Neste trabalho são utilizados dados provenientes da missão GEOSAT (ERM) (GEOdetic SATellite - Exact Repeat Mission) mostrados na Fig. 2. Os dados brutos apresentam registros com informações sobre a altitude da superfície oceânica em relação ao elipsóide de referência em duas formas: um valor médio amostrado com intervalo de 1 segundo, e valores pontuais em intervalos de $1 / 10$ segundo. Além disso, os registros contêm correções e parâmetros necessários para se obter a altitude instantânea da superfície oceânica em relação ao elipsóide de referência (sea surface height - SSH).

As correções devem ser aplicadas aos dados de satélite a fim de eliminar diversos efeitos que afetam os valores medidos pelo altímetro (Cheney et al., 1987), além do cálculo de rotas médias devido à variação sazonal de SSH. Os dados de satélite também apresentam diferenças de cruzamento que devem ser analisadas e ajustadas, através de correções aplicadas a cada rota, de modo a homogeneizar o conjunto de dados altimétricos a serem utilizados no processamento.

\section{METODOLOGIA}

\section{SELEÇÃO E AJUSTAMENTO DOS DADOS}

\section{Seleção dos dados de gravimetria marinha}

Antes de iniciar o ajustamento, deve-se eliminar os dados que contém erros grosseiros de forma a garantir um boa distribuição dos valores de anomalia na região. Isto é feito admitindo um limite máximo permitido para a amplitude relativa das anomalias gravimétricas. Um limite de $\pm 50 \mathrm{mGal}$ foi considerado razoável de acordo com a distribuição global dos dados. Quando possível, compararam-se as rotas gravimétricas com os demais dados geofísicos disponíveis, a fim de detectar também deslocamentos sistemáticos. Os dados de gravimetria marinha originais e aqueles selecionados segundo estes critérios estão apresentados na Fig. 3. Dos 120 cruzeiros armazenados inicialmente, 93 foram selecionados e utilizados no ajustamento, originando 36.854 pontos de gravimetria marinha.

\section{Diferenças de cruzamento}

Os pontos onde ocorrem cruzamentos das rotas de um cruzeiro marítimo são denominados pontos de cruzamento. A diferença entre os valores correspondentes a esses pontos é denominada diferença de cruzamento interno (dci) quando o cruzamento ocorre entre rotas de um mesmo cruzeiro e, diferença de cruzamento externo (dce), quando o cruzamento ocorre entre rotas de cruzeiros diferentes. Assim, é necessário realizar ajustamentos que minimizem essas diferenças, visando melhorar a consistência dos dados e estimar sua precisão global. As diferenças de cruzamento podem provocar o aparecimento de feições artificiais na representação do campo de gravidade. Os termos dci e dce também serão utilizados para denotar as diferenças de cruzamento existente entre os dados de altimetria por satélite.

\section{Ajustamento dos dados de gravimetria marinha}

No caso da gravimetria marinha, os principais fatores que podem influenciar nas diferenças de cruzamento são (Wessel \& Watts, 1988):

1) sensibilidade do equipamento de medição;

2) erros causados por problemas no nivelamento do equipamento; 
3) deriva instrumental;

4) realização inadequada, ou não realização, da abertura e/ou fechamento da linha nas estações básicas, e

5) posicionamento incorreto do navio, levando à localização dos pontos e correção de Eötvös inadequada.

As dce permitem identificar os erros associados aos fatores (3) e (4). A correção de Eötvös é dada pela expressão (Dehlinger, 1978):

$$
\mathrm{E}=14,585 \mathrm{v} \cos \phi \sin \alpha+0,015696 \mathrm{v}^{2},
$$

onde $\mathrm{v}$ é a velocidade do navio em $\mathrm{m} / \mathrm{s}, \alpha$ é o azimute do navio contado a partir do norte no ponto em questão e $\phi$ é a latitude geográfica do ponto. Pode-se notar que um posicionamento inadequado conduz a um valor de $\alpha$ incorreto. Para analisar e corrigir este efeito, devese calcular as dci, considerando cada rota como independente das demais, e efetuar o ajuste para essas diferenças de forma a minimizar a soma do quadrado das mesmas.

O método de busca dos pontos de cruzamento (Prince \& Forsyth, 1984) consiste em dividir a região de interesse em blocos de tamanhos adequados e determinar, em cada bloco, os cruzeiros que contém pontos presentes na área. Assim, tem-se uma lista com os blocos identificados, por exemplo, pelas coordenadas do extremo inferior esquerdo, e os cruzeiros que têm possibilidade de apresentar pontos de cruzamento entre si. Pode-se, então, obter as combinações de pares de cruzeiros para a análise.

As dce são ajustadas utilizando um modelo linear $\mathbf{y}=a \mathbf{x}+b$ para representar essas diferenças. Determina-se então o parâmetro $a$ para corrigir o efeito da deriva e o parâmetro $b$ para o deslocamento. Após aplicar estas correções aos dados, nota-se que as dce ainda existem. Isto ocorre, na maioria dos casos, devido ao posicionamento inadequado do navio e pode ser corrigido analisando-se as dci (Prince \& Forsyth, 1984). Este procedimento permite detectar rotas isoladas, que apresentam deslocamento em relação às demais, e calcular correções para as mesmas de modo a minimizar a soma do quadrado das dei de todas as rotas de um mesmo cruzeiro.

Com as correções efetuadas foi possível constatar uma melhora significativa nos parâmetros estatísticos referentes a esses dados, assegurando uma maior consistência em todo o conjunto e permitindo a estimativa da precisão global. A Fig. 4 apresenta a distribuição das diferenças após o ajustamento.

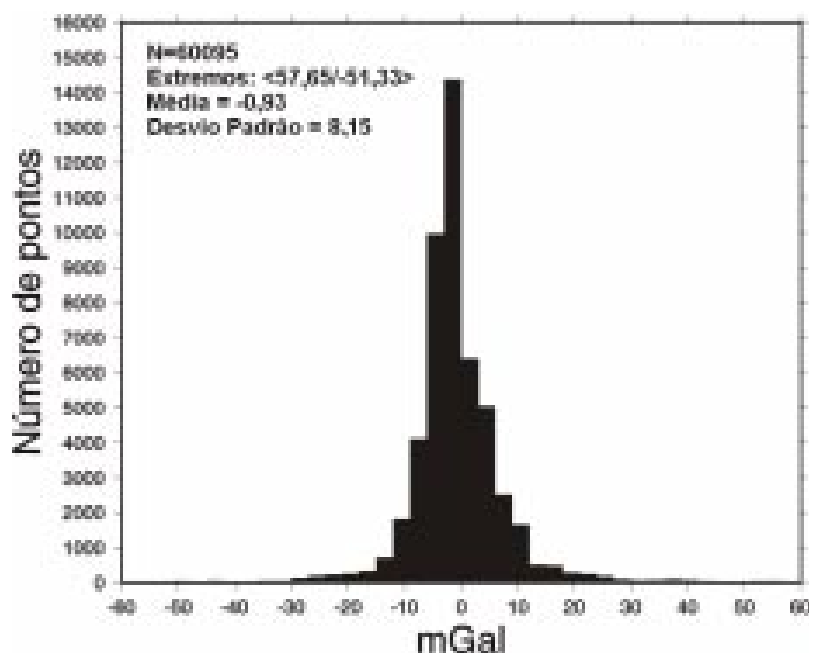

Figura 4 - Histograma das diferenças de cruzamento após o ajuste.

Figure 4 - Cross-over errors hystogram after the adjustment.

\section{Seleção dos dados altimétricos}

Para a seleção dos dados altimétricos, é feita uma análise da qualidade do sinal recebido pelo satélite (Sandwell \& McAdoo, 1990). São estabelecidos limites superiores para o desvio padrão em cada conjunto de 10 pontos amostrados em 1/10 segundo para comprimento de onda significativo e um intervalo para o ganho automático. Pontos com indicação de estarem sobre terra ou gelo também são descartados.

Depois deste pré-processamento, parte-se para a detecção de dados com erros grosseiros, utilizando-se o seguinte critério:

- o valor de SSH para cada ponto é comparado com o valor da altura geoidal fornecida pelo modelo geopotencial OSU89 (Rapp \& Pavlis, 1990); pontos com diferenças maiores do que 20 metros são eliminados;

- pontos cujo valor de SSH, em módulo, ultrapassou 100 m são também descartados.

A etapa final é calcular as rotas médias que representem um conjunto de rotas coincidentes ao longo do período de 1 ano, onde as medidas são afetadas pela variação sazonal. Um valor típico de variação máxima devido a efeitos sazonais é de $0,5 \mathrm{~m}$. Isto permite melhorar a precisão dos dados, atenuando os erros de órbita que podem atingir até dezenas de centímetros, sem que a distribuição geográfica original dos dados seja modificada significativamente.

Após a aplicação das correções pertinentes verifica-se que a SSH apresenta variação máxima de 2,0 m em módulo e, dentro da precisão de 1 a 2 m, a SSH 
pode ser considerada a altura geoidal. A diferença entre a altura geoidal e a SSH fornece a topografia dinâmica da superfície oceânica (sea surface topography-SST), que pode ser utilizada para estudos oceanográficos e meteorológicos.

Com este procedimento obtiveram-se os dados de altimetria por satélite utilizados neste trabalho, totalizando 10.435 pontos, com espaçamento médio de 7 $\mathrm{km}$ ao longo das rotas, e de aproximadamente $150 \mathrm{~km}$, entre as rotas.

\section{Ajustamento dos dados altimétricos}

As diferenças de cruzamento ocorrem principalmente devido a erros associados ao cálculo da órbita do satélite e imprecisões na estimativa dos parâmetros de correções aos dados. A análise dessas diferenças é necessária para fornecer a precisão global do conjunto de dados e para verificar a existência de erros sistemáticos nas rotas.

Para calcular as correções a serem aplicadas a cada rota, de modo a minimizar a soma do quadrado das diferenças de cruzamento entre as mesmas, é necessário admitir que a principal causa dessas diferenças está associada aos erros de órbita.

Desta forma, o modelo utilizado para este ajuste pelo método dos mínimos quadrados admite que:

a) os valores de $\mathrm{SSH}$ interpolados nos pontos de cruzamento são corretos; e

b) independentemente das causas dos erros de órbita, elas permanecem constantes ao longo da rota considerada.

Assim, o problema resume-se em resolver um sistema de equações lineares, onde as diferenças da SSH entre as rotas são as observações e as correções a serem aplicadas a cada rota são os parâmetros a serem determinados.

Neste método, é necessário fixar uma das rotas e calcular as correções das demais em relação a ela. Fixando-se cada uma das rotas pode-se determinar aquela que proporcionou distribuição com média e resíduo quadrático médio menores, sendo esta a solução final para o cálculo das correções a serem aplicadas às rotas médias.

O resíduo quadrático médio das diferenças de cruzamento das rotas após o ajuste é de $0,11 \mathrm{~m}$, que pode ser adotado como representativo da precisão média global deste conjunto de dados.

\section{ESTIMAÇÃO DOS ELEMENTOS DO CAM- PO DE GRAVIDADE}

\section{Elementos do campo de gravidade utilizados neste trabalho}

Os elementos do campo utilizados neste trabalho podem ser expressos através de funcionais lineares aplicados ao potencial anômalo (T). A fórmula de Bruns relaciona a altura geoidal $(\mathrm{N})$ com o potencial anômalo:

$$
\mathrm{N}=\frac{\mathrm{T}}{\gamma}
$$

onde $\gamma$ é a aceleração da gravidade sobre o elipsóide de referência. A equação fundamental da Geodésia Física (Heiskanen \& Moritz, 1967) relaciona a anomalia ar-livre $(\Delta \mathrm{g})$ com o potencial anômalo:

$$
\Delta \mathrm{g}=-\frac{\partial \mathrm{T}}{\partial \mathrm{r}}-\frac{2 \mathrm{~T}}{\mathrm{r}}
$$

onde r é a distância entre o centro de massa da Terra e um ponto na sua superfície.

A representação do campo de gravidade terrestre consiste em determinar uma função aproximante ao potencial anômalo, a partir dos dados disponíveis e, através desta, calcular os diversos elementos associados ao campo gravitacional (e.g., anomalia ar-livre e altura geoidal).

O potencial anômalo é uma função harmônica no exterior das massas atrativas. Assim, o funcional expresso pela fórmula de Bruns na Eq. (2) representa, na verdade, a anomalia de altitude, que coincide com a altura geoidal na região oceânica. No presente trabalho utilizaremos o termo altura geoidal para este funcional.

\section{Colocação por mínimos quadrados}

A colocação por mínimos quadrados (Moritz, 1980) pode ser utilizada quando o problema da representação do campo de gravidade envolve dados heterogêneos em tipo e precisão. A aplicação desta técnica no presente trabalho consiste basicamente em:

- estimar uma função aproximante ao potencial anômalo $(\hat{T})$ a partir de funcionais lineares dos elementos envolvidos (neste caso, $\mathrm{L}_{\Delta \mathrm{g}}$ e $\mathrm{L}_{\mathrm{N}}$ ) aplicados a $\mathbf{T}$, e - estimar os elementos do campo, $\Delta \hat{\mathrm{g}}$ e $\hat{\mathrm{N}}$ através dos respectivos funcionais lineares $\mathrm{L}_{\Delta \mathrm{g}}$ e $\mathrm{L}_{\mathrm{N}}$ aplicados a $\hat{T}$.

Adotando a terminologia de Moritz (1980), um 
conjunto de observações $\mathbf{I}$ associadas a um elemento genérico do campo de gravidade, pode ser expresso como um funcional linear (L) aplicado ao potencial anômalo (T) na forma:

$$
\mathbf{l}=\mathrm{LT} \text {. }
$$

Como em geral as observações são afetadas por erros aleatórios $(\mathbf{n})$, resulta:

$$
\mathbf{l}=\mathrm{LT}+\mathbf{n} \text {. }
$$

Para generalizar o modelo, consideram-se os parâmetros funcionais não aleatórios como um vetor $\mathbf{X}$, tal que o sinal (LT) seja representado por uma componente determinística $\mathbf{A X}$, onde $\mathbf{A}$ é a matriz de sensibilidade, e uma componente estocástica BT, onde B é o vetor que contém os funcionais lineares dos elementos do campo gravitacional envolvidos. Assim sendo, obtém-se o modelo geral para o conjunto de observações:

$$
\mathbf{l}=\mathbf{A X}+\mathbf{B} \mathbf{T}+\mathbf{n} .
$$

Supondo a condição de unicidade da solução, temse:

$$
\boldsymbol{\alpha}<\mathbf{T}, \mathbf{T}>+\boldsymbol{\beta}<\mathbf{n}, \mathbf{n}>=\text { mínimo, }
$$

o modelo tem solução analítica dada por Sá (1988), onde:

$$
\mathbf{X}=\left[\mathbf{A}^{\mathbf{T}}(\beta \mathbf{C}+\alpha \mathbf{D})^{-1} \mathbf{A}\right] \mathbf{A}^{\mathbf{T}}(\beta \mathbf{C}+\alpha \mathbf{D})^{-1} \mathbf{l} .
$$

e

$$
\mathbf{T}=(\mathbf{B K})^{\mathbf{T}}(\beta \mathbf{C}+\alpha \mathbf{D})^{-1}(\mathbf{l}+\mathbf{A X}) .
$$

onde $<$,> representa o produto interno, $\alpha$ e $\beta$ são parâmetros numéricos, $\mathbf{K}$ é a aproximação discreta para o núcleo do espaço de Hilbert, $\mathbf{C}$ e $\mathbf{D}$ são matrizes simétricas definidas positivas. Conforme os valores adotados para os parâmetros, para o núcleo e para as matrizes, a solução geral pode ter formas particulares admitindo interpretações distintas.

A colocação por mínimos quadrados constitui um caso particular em que $\mathbf{T}$ é considerado um processo aleatório, ou seja, as quantidades envolvidas devem oscilar em torno da média.

As variâncias associadas aos parâmetros $\left(\sigma_{x}^{2}\right)$ e ao potencial anômalo $\left(\sigma_{\mathrm{T}}^{2}\right)$ devem ser minimizadas. Para isso, deve-se adotar valores unitários para a e b e utilizar a função covariância do sinal para representar o núcleo do espaço de Hilbert. Desta forma, a solução para o vetor dos sinais é:

$$
\mathbf{s}=\mathbf{C}_{\mathrm{sl}} \mathbf{C}_{11}^{-1}(\mathbf{l}-\mathbf{A X})
$$

e para o vetor das variâncias

$$
\boldsymbol{\sigma}_{\mathrm{s}}=\mathbf{C}_{\mathrm{ss}}-\mathbf{C}_{\mathrm{sl}} \mathbf{C}_{\mathrm{ll}}{ }^{-1} \mathbf{C}_{\mathrm{ls}},
$$

onde $\mathbf{C}_{\mathrm{ss}}$ é a matriz covariância do sinal, $\mathbf{C}_{11}$ é a matriz covariância dos valores observados e $\mathbf{C}_{\mathrm{sl}}$ é a matriz covariância entre o sinal e a observação.

Como o cálculo de covariâncias envolve uma quantidade muito grande de dados, calculam-se covariâncias amostrais, que representam a região de estudo, e a elas ajustam-se modelos matemáticos que permitem obter as covariâncias para toda a área. Essas matrizes são preenchidas a partir destes modelos ajustados.

\section{Cálculo e representação de covariâncias}

Do ponto de vista teórico, qualquer tipo de dado associado ao potencial anômalo pode ser utilizado para calcular as covariâncias necessárias. A anomalia arlivre é a mais utilizada para este fim porque apresenta uma distribuição geográfica mais homogênea e são dados disponíveis em maior quantidade.

Representando a anomalia observada pelas componentes determinística $\left(\Delta \mathrm{g}^{\mathrm{d}}\right)$ e estocástica $\left(\Delta \mathrm{g}^{\mathrm{r}}\right)$, temos:

$$
\Delta \mathrm{g}=\Delta \mathrm{g}^{\mathrm{d}}+\Delta \mathrm{g}^{\mathrm{r}}
$$

onde a componente determinística é dada pela equação fundamental da Geodésia Física, considerando aproximação esférica (Eq. (3)). Dado um conjunto de componentes estocásticas da anomalia ar-livre, temse:

$$
\Delta g^{r}=\left\{\Delta g_{1}^{r}, \Delta g_{2}^{r}, \ldots, \Delta g_{n}^{r}\right\}
$$

e as covariâncias entre esses elementos são dadas pelo operador média, tal que:

$$
\mathrm{C}(\Psi)=\operatorname{cov}\left(\Delta \mathrm{g}_{\mathrm{i}}^{\mathrm{r}}, \Delta \mathrm{g}_{\mathrm{j}}^{\mathrm{r}}\right)=\mathrm{M}\left\{\Delta \mathrm{g}_{\mathrm{i}}^{\mathrm{r}} \cdot \Delta \mathrm{g}_{\mathrm{j}}^{\mathrm{r}}\right\},
$$

sendo $\Psi$ a distância esférica entre as anomalias $\Delta \mathrm{g}_{\mathrm{i}} \mathrm{e}$ $\Delta \mathrm{g}_{\mathrm{j}}$ e $\mathrm{M}\{$.$\} é o operador média.$

Nota-se que a covariância indica a correlação estatística entre as grandezas, no caso as anomalias ar-livre. Desta forma, esta quantidade mostra a tendência das anomalias possuírem mesmo sinal e amplitude. As anomalias não são correlacionadas quando a covariância é igual a zero. Na prática as covariâncias são calculadas pela expressão:

$$
\mathrm{C}\left(\Psi_{\mathrm{k}}\right)=\frac{1}{\mathrm{n}_{\mathrm{k}}} \sum_{\mathrm{i}=1}^{\mathrm{n}} \sum_{\mathrm{j}=1}^{\mathrm{n}} \Delta \mathrm{g}_{\mathrm{i}}^{\mathrm{r}} \Delta \mathrm{g}_{\mathrm{j}}^{\mathrm{r}},
$$

onde k é o índice de classificação segundo distâncias. Deste modo, $\mathrm{C}\left(\Psi_{\mathrm{k}}\right)$ é a média dos produtos dos elementos existentes em pontos que estão à distância $\Psi_{\mathrm{ij}}$, tal que:

$$
\Psi_{\mathrm{k}}-\frac{\Delta \Psi}{2} \leq \Psi_{\mathrm{ij}} \leq \Psi_{\mathrm{k}}+\frac{\Delta \Psi}{2}
$$

e $\Delta \Psi$ é o intervalo de distância das classes. Desta maneira, é necessário pré-estabelecer $\Delta \Psi$ e a quantidade de intervalos utilizados. Este procedimento proporciona rapidez no processamento sem perda sig- 
nificativa de informação nos resultados.

A Eq.(15) pressupõe que as covariâncias são isotrópicas, ou seja, as covariâncias não devem depender da direção mas somente da distância considerada. Na prática, deve-se estabelecer uma distância limite para qual pode-se utilizar covariâncias isotrópicas. Para isso obtém-se os gráficos das covariâncias calculadas bidimensionalmente, e observa-se a máxima distância para qual o gráfico apresenta simetria em relação ao eixo das covariâncias (Sá, 1988). Além disso, as covariâncias devem apresentar homogeneidade, o que pode ser constatado através do cálculo e análise das mesmas para sub-regiões distintas dentro da região de estudo.

\section{Funções covariâncias}

Como o cálculo de covariâncias pelo processo de amostragem é lento devido à quantidade e distribuição irregular dos pontos, ele é efetuado somente para a obtenção de covariâncias amostrais, às quais são ajustados modelos matemáticos próprios para a colocação por mínimos quadrados, denominados funções covariâncias. Quando as covariâncias são homogêneas e isotrópicas os modelos são relativamente simples, sendo caracterizados por três parâmetros fundamentais: o valor que a função assume em $\Psi=0$ (variância):

$$
\mathrm{C}_{0}=\mathrm{C}(0) \text {, }
$$

a distância $\xi$ para a qual a covariância é igual a metade do valor da variância (distância de correlação)

$$
\mathrm{C}(\xi)=\frac{\mathrm{C}_{0}}{2} .
$$

O parâmetro de curvatura, associado à curvatura da função covariância em $\Psi=0$ que é dado por:

$$
\chi=\kappa \frac{\xi^{2}}{C_{0}},
$$

sendo $\kappa \mathrm{o}$ valor da curvatura. A expressão envolvendo a variância do gradiente horizontal da anomalia ar-livre $\mathrm{G}_{0}$ é mais conveniente para calcular este parâmetro, sendo:

$$
\chi=\xi^{2} \frac{\mathrm{C}_{0}}{\mathrm{G}_{0}} .
$$

A função covariância deve reproduzir as características estatísticas dos dados e admitir uma representação matemática simples. Os modelos utilizados podem ser classificados em globais ou locais. Os modelos globais são calculados a partir de valores médios de gravimetria convencional médios e de da- dos de satélites artificiais. Nos modelos locais, calculamse as covariâncias a partir de dados gravimétricos pontuais, após a remoção das componentes determinísticas que representam características globais.

\section{Funções covariâncias esféricas}

Desenvolvendo a Eq. (3) em harmônicos esféricos para o exterior da esfera, a função covariância da anomalia ar-livre entre os pontos $\mathrm{P}$ e $\mathrm{Q}$, situados a distância $\Psi$, tem a forma (Sá, 1988):

$$
\mathrm{C}(\mathrm{P}, \mathrm{Q})=\sum_{\mathrm{n}=2}^{\infty} \sigma_{\mathrm{n}}(\Delta \mathrm{g}, \Delta \mathrm{g})\left(\frac{\mathrm{R}_{\mathrm{B}}^{2}}{\mathrm{r}_{\mathrm{P}} \mathrm{r}_{\mathrm{Q}}}\right)^{\mathrm{n}+2} \mathrm{P}_{\mathrm{n}}(\cos \Psi),
$$

onde $\mathrm{R}_{B}$ é o raio da maior esfera totalmente contida na Terra, denominada esfera de Bjerhammar, $r_{P}$ e $r_{Q}$ são os raios vetores dos pontos $\mathrm{P}$ e $\mathrm{Q}$, e $\sigma_{\mathrm{n}}(\Delta \mathrm{g}, \Delta \mathrm{g})$ é a variância de grau da anomalia ar-livre, dada por:

$$
\sigma_{n}(\Delta \mathrm{g}, \Delta \mathrm{g})=\mathrm{M}\{\Delta \mathrm{g} \cdot \Delta \mathrm{g}\} \text {. }
$$

Para que seja possível generalizar esta metodologia para qualquer elemento do campo gravitacional da Terra é necessário determinar também a função covariância do potencial anômalo. Para isso, utiliza-se a relação entre a anomalia ar-livre e o potencial anômalo para um mesmo grau do desenvolvimento em harmônicos esféricos, como:

$$
\mathrm{T}_{\mathrm{n}}=\frac{\mathrm{R}_{\mathrm{B}}}{\mathrm{n}-1} \Delta \mathrm{g}_{\mathrm{n}} .
$$

Desta forma, a relação entre a variância de grau é dada por:

$$
\sigma_{\mathrm{n}}(\mathrm{T}, \mathrm{T})=\left(\frac{\mathrm{R}_{\mathrm{B}}}{\mathrm{n}-1}\right)^{2} \sigma_{\mathrm{n}}(\Delta \mathrm{g}, \Delta \mathrm{g}) .
$$

obtendo para a função covariância do potencial anômalo a expressão:

$$
\begin{aligned}
K(P, Q)=\sum_{n=2}^{\infty}\{ & \left(\frac{R_{B}}{n-1}\right)^{2} \sigma_{n}(\Delta g, \Delta g) \\
& \left.\left(\frac{R_{B}^{2}}{r_{P} r_{Q}}\right)^{n+2} P_{n}(\cos \Psi)\right\} .
\end{aligned}
$$

Utilizando a propagação das covariâncias (Moritz, 1980), é possível obter, a partir da Eq. (25), as covariâncias entre quaisquer elementos relacionados teoricamente ao potencial anômalo através da expressão abaixo:

$$
\operatorname{cov}\left(\mathrm{L}_{\mathrm{i}}(\mathrm{P}), \mathrm{L}_{\mathrm{j}}(\mathrm{Q})\right)=\mathrm{L}_{\mathrm{i}}(\mathrm{P}) \mathrm{L}_{\mathrm{j}}(\mathrm{Q}) \mathrm{K}(\mathrm{P}, \mathrm{Q}),
$$




\begin{tabular}{|c|c|c|}
\hline $\mathrm{A}\left(\mathrm{mGal}^{2}\right)$ & $\mathbf{R}_{\mathrm{B}}-\mathbf{R}(\mathrm{m})$ & $\mathbf{s}$ \\
\hline 214,49 & $-5541,2$ & 0,99913 \\
\hline
\end{tabular}

Tabela 1 - Parâmetros calculados pelo ajustamento da covariância amostral da anomalia ar-livre.

Table 1 - Calculated parameters adjusting the covariances offree-air anomalies.

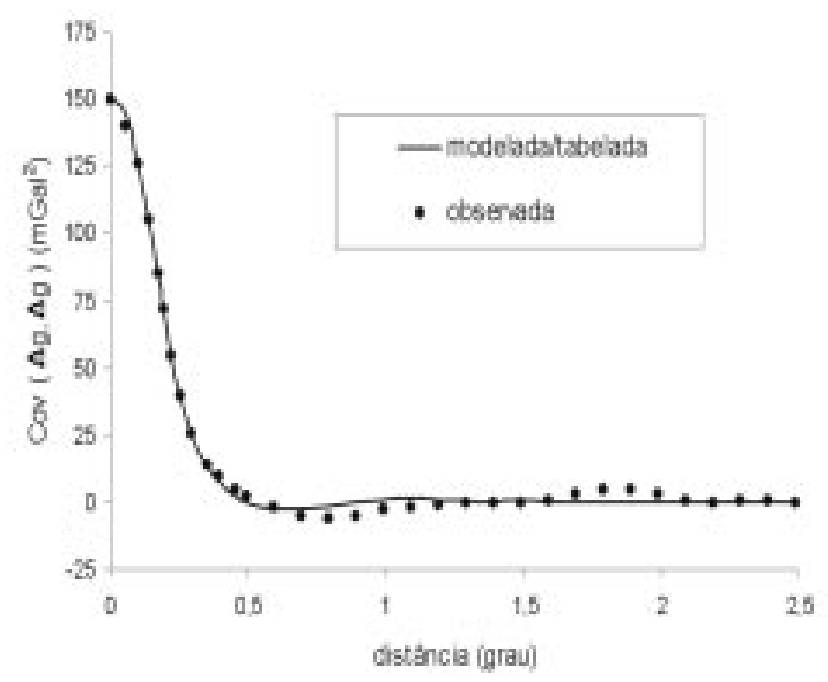

Figura 5 - Covariâncias amostrais da anomalia ar-livre obtidas a partir do modelo digital de Sandwell \& Yale (1994) e a função covariância ajustada.

Figure 5 - Covariances of the free-air anomaly obtained from digital model of Sandwell \& Yale (1994) and the adjusted covariance function.

onde i e j representam genericamente $\Delta \mathrm{g}$ e $\mathrm{N}$.

Como as expressões envolvem somas infinitas, deve-se obter modelos que possam representá-las através da soma de termos finitos. A técnica da decomposição, utilizada para solucionar este problema, consiste em representar as funções através de funções auxiliares que possuam um número finito de termos e suas derivadas. Antes disso, deve-se adotar um modelo que represente a variância de grau na Eq. (25). O modelo proposto por Tscherning \& Rapp (1974) é o mais utilizado para este fim e é dado por:

$$
\sigma_{\mathrm{n}}(\Delta \mathrm{g}, \Delta \mathrm{g})=\frac{\mathrm{A}(\mathrm{n}-1)}{(\mathrm{n}-2)(\mathrm{n}+\mathrm{B})} \quad \mathrm{n}>2,
$$

onde A e B são parâmetros a determinar. Desta forma, a expressão com termos finitos que permite calcular a covariância da anomalia ar-livre entre dois pontos $\mathrm{P}$ e $\mathrm{Q}$ situados a uma distância $\Psi$ entre si, é dada por (Sá, 1988):

$$
\begin{aligned}
\operatorname{cov}\left(\Delta g_{P}, \Delta g_{Q}\right)=\frac{A s}{B+2} & {\left[( B + 1 ) \left(F_{B}-\frac{s}{B}-\frac{s^{2} t}{B+1}-\right.\right.} \\
& \left.\left.-\frac{s^{3} P_{2}(t)}{B+2}\right)+F_{-2}\right]
\end{aligned}
$$

onde

$$
\mathrm{t}=\cos \Psi
$$

e

$$
\mathrm{s}=\left(\frac{\mathrm{R}_{\mathrm{B}}^{2}}{\mathrm{r}_{\mathrm{P}} \mathrm{r}_{\mathrm{Q}}}\right),
$$

as funções $\mathrm{F}_{\mathrm{B}}$ e $\mathrm{F}_{-2}$ são funções auxiliares dependentes de s e t (Sá, 1988). Para as covariâncias do potencial anômalo tem-se:

$$
\begin{aligned}
\operatorname{cov}\left(T_{P}, T_{Q}\right) & =\frac{A R_{B}^{2}}{(B+2)(B+1)}+\left[(B+1) F_{-2}-(B+2)\left(F_{-1}-s^{2} P(t)\right)-\frac{s}{B}-\right. \\
& \left.-\frac{s^{2} t}{B+1}-\frac{s^{2} P_{2}(t)}{B+2}\right]
\end{aligned}
$$

Utilizando a propagação, as covariâncias da altura geoidal são calculadas por:

$$
\begin{aligned}
\operatorname{cov}\left(N_{P}, N_{Q}\right)= & \frac{1}{\gamma_{p} \gamma_{Q}}\left\{\frac{A R_{B}^{2}}{(B+2)(B+1)}+\left[(B+1) F_{-2}-(B+2)\left(F_{-1}-s^{3} P(t)\right)-\frac{s}{B}\right.\right. \\
& \left.\left.-\frac{s^{2} t}{B+1}-\frac{s^{3} P_{2}(t)}{B+2}\right]\right\}
\end{aligned}
$$

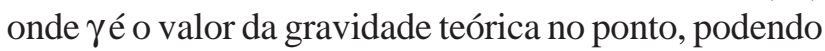
ser substituída pelo valor da aceleração da gravidade média na superfície. No caso das covariâncias entre a anomalia ar-livre e a altura geoidal, obtém-se a expressão:

$$
\begin{aligned}
& \operatorname{cov}\left(\Delta g_{P}, N_{Q}\right)=\frac{A R_{B}^{2}}{r_{P} \gamma_{Q}(B+2)}+ \\
& +\left[F_{-2}-F_{-1}-\frac{s^{2} t}{B+1}-\frac{s^{3} P_{2}(t)}{B+2}\right],
\end{aligned}
$$

As Eqs. (28), (32) e (33) permitem preencher respectivamente as matrizes $\mathbf{C}_{\mathrm{ss}}, \mathbf{C}_{11}$ e $\mathbf{C}_{\mathrm{sl}}$.

$\mathrm{Na}$ prática, adota-se a aproximação esférica de forma que:

$$
\mathrm{r}_{\mathrm{P}}=\mathrm{r}_{\mathrm{Q}}=\mathrm{R}
$$

onde $\mathrm{R}$ é o raio terrestre médio.

Desta forma, tem-se todas as grandezas necessárias para estimar $\Delta \mathrm{g}$ e $\mathrm{N}$ a partir das Eqs. (10) e (11). 


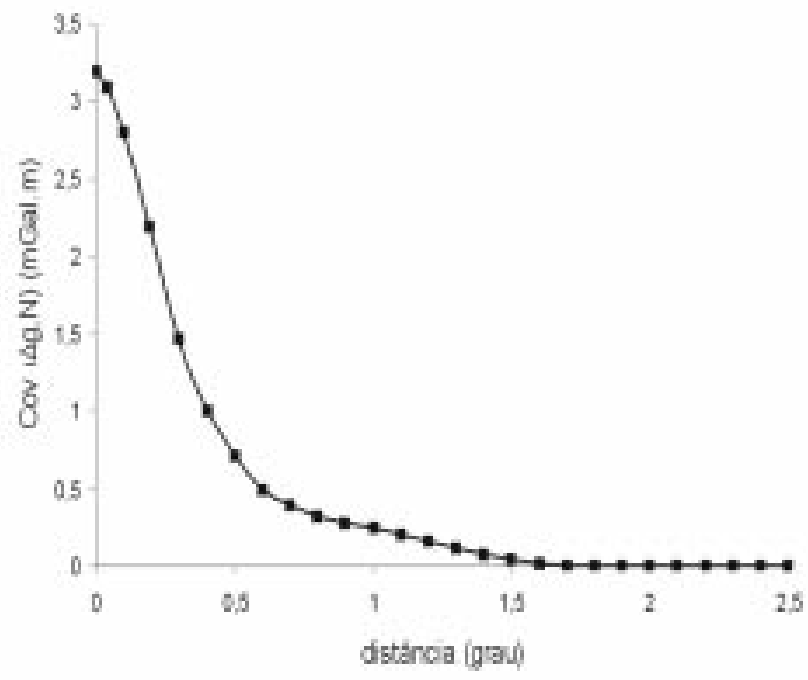

Figura 6 - Covariâncias entre anomalia ar-livre e altura geoidal obtidas através da propagação das covariâncias da anomalia ar-livre.

Figure 6 - Covariances between free-air anomaly and geoid heights obtained from the propagation of the free-air anomaly covariances.

\begin{tabular}{|c|c|c|}
\hline Elementos & $\mathbf{C}_{\mathbf{0}}$ & $\boldsymbol{\xi}$ (graus decimais) \\
\hline $\operatorname{Cov}(\Delta \mathrm{g}, \Delta \mathrm{g})$ & $151,64 \mathrm{mGal}^{2}$ & 0,176 \\
\hline $\operatorname{cov}(\Delta \mathrm{g}, \mathrm{N})$ & $3,1862 \mathrm{mGal} . \mathrm{m}$ & 0,295 \\
\hline $\operatorname{cov}(\mathrm{N}, \mathrm{N})$ & $0,1366 \mathrm{~m}^{2}$ & 0,668 \\
\hline
\end{tabular}

Tabela 2 - Parâmetros essenciais das funções covariância entre os elementos do campo de gravidade utilizados neste trabalho.

Table 2 - Main parameters of covariance functions between the gravity field elements used in this study.

\section{Ajustamento das covariâncias amostrais}

Os parâmetros essenciais da função covariância definem características locais. As variáveis relacionadas a esses parâmetros, presentes nas fórmulas finitas, são: $\mathrm{B}, \mathrm{A}$ e $\mathrm{R}_{\mathrm{B}}$.

A variável B descreve a estrutura da variância de grau e não pode ser obtida a partir de dados locais. Assim, adotou-se um valor obtido através de dados globais ( $\mathrm{B}=24)$ para esta variável (Tscherning \& Rapp, 1974). Os valores de $R_{B}$ e $A$ podem ser determinados ajustando-se um modelo através do método dos mínimos quadrados onde os parâmetros a serem determinados correspondem a estas variáveis. Tratando-se de um modelo não linear em relação $a R_{B}$, pode-se linearizálo e encontrar a solução iterativamente. Este método é descrito em Knudsen (1987).

Para cada iteração, a determinação dos parâmetros é dada por:

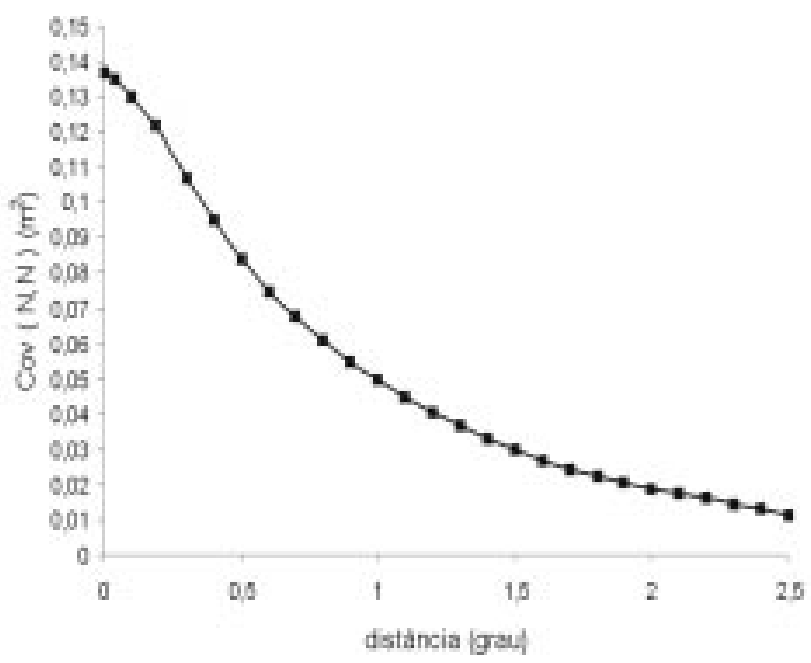

Figura 7 - Covariâncias entre alturas geoidais obtidas através da propagação das covariâncias da anomalia ar-livre.

Figure 7 - Covariances between geoid heights obtained from the propagation of the free-air anomaly covariances.

$$
\mathbf{x}-\mathbf{x}_{0}=\left(\mathbf{J}^{\mathbf{T}} \mathbf{C}_{\mathbf{y}}^{-1} \mathbf{J}+\mathbf{C}_{\mathbf{x}}^{-1}\right)^{-1} \mathbf{J}^{\mathbf{T}} \mathbf{C}_{\mathbf{y}}^{-1}\left(\mathbf{y}-\mathbf{y}_{0}\right)
$$

onde $\mathbf{x}$ é o vetor de parâmetros ajustados a partir dos valores dados por $\mathbf{x}_{\mathbf{0}} ; \mathbf{y}$ contém os valores das covariâncias empíricas; $\mathbf{y}_{0}$ contém os valores do modelo para $\mathbf{x}=\mathbf{x} ; \mathbf{J}$ é a matriz Jacobiano contendo os valores de $\frac{\partial \mathrm{y}_{\mathrm{i}}}{\partial \mathrm{x}_{\mathrm{j}}} ; \mathbf{C}_{\mathrm{y}}$ é a matriz dos erros de $\mathbf{y}$, e $\mathbf{C}_{\mathrm{x}}$ é a matriz de variância de $\left(\mathbf{x}-\mathbf{x}_{\mathbf{0}}\right)$.

Adotando os valores iniciais $\left(\mathbf{x}_{\mathbf{0}}\right)$ para o modelo determina-se o conjunto de parâmetros $(\mathbf{x})$ iterativamente.

A vantagem deste método é que ele pode utilizar como dados de entrada as covariâncias de qualquer número de elementos associados ao campo de gravidade e, a partir destes, determinar o melhor conjunto de parâmetros através da combinação dos dados. No presente trabalho foram utilizados somente valores de anomalia ar-livre para este ajustamento.

\section{RESULTADOS}

\section{Parâmetros da função covariância}

A verificação da isotropia, através do cálculo de covariâncias unidimensionais, mostrou que para distâncias de até $0,5^{\circ}$ as covariâncias podem ser consideradas isotrópicas. A análise em conjunto das covariâncias unidimensionais calculadas em blocos de 
$5^{\circ} \times 5^{\circ}$ comprova a homogeneidade desta grandeza na região de estudo.

O ajuste das covariâncias amostrais calculados através da Eq. (28), resultou nos parâmetros apresentados na Tab. 1.

A Fig. 5 mostra as covariâncias amostrais obtidas a partir do modelo digital de anomalia ar-livre de Sandwell \& Yale (1994) e a função ajustada às mesmas pelo método descrito em Knudsen (1987).

Dispondo destes parâmetros pode-se calcular as covariâncias entre os demais elementos do campo e as covariâncias cruzadas, utilizando respectivamente as expressões (32) e (33). As Figs. 6 e 7 apresentam as funções assim calculadas para as covariâncias entre a anomalia ar-livre e altura geoidal $\mathrm{C}(\Delta \mathrm{g}, \mathrm{N})$ e entre a altura geoidal $\mathrm{C}(\mathrm{N}, \mathrm{N})$. Os parâmetros essenciais para as três funções covariâncias envolvidas neste caso encontram-se na Tab. 2.

\section{Representação integrada do campo de gravi- dade}

A vantagem de se representar o campo gravimétrico utilizando dados de altimetria por satélite combinados a dados de gravimetria marinha reside na possibilidade de se aproveitar toda a informação referente a este campo, permitindo a representação de detalhe onde a densidade de dados marinhos é elevada. Modelos digitais do campo de gravidade obtidos utilizando apenas dados de altimetria por satélite apresentam um alto nível de ruído devido ao procedimento para o ajuste das rotas e inversão dos dados (Neumann et al., 1993). Além disso, existe uma perda de resolução determinística dos dados de satélite nas áreas próximas ao continente (Molina \& Sá, 1993).

Para a representação integrada foram utilizados

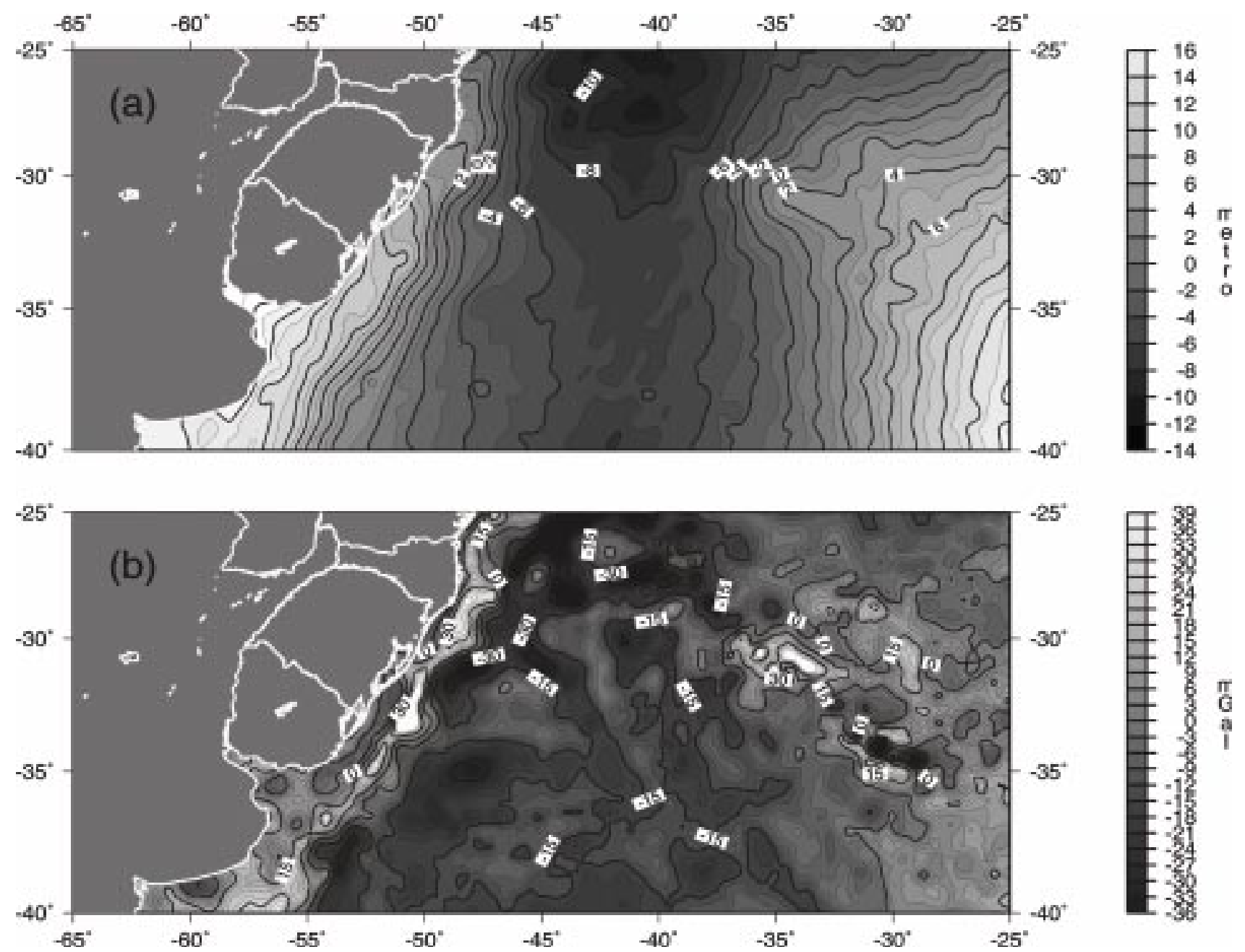

Figura 8 - Modelos do campo gravitacional: (a) altura geoidal, intervalo de contorno: $1 \mathrm{~m}$; intervalo da gradação em cinza: $2 \mathrm{~m}$ (b) anomalia ar-livre, intervalo de contorno: $15 \mathrm{mGal}$ e intervalo da gradação em cinza: $3 \mathrm{mGal}$.

Figure 8 - Gravity field models: (a) geoid heights, contour interval $1 \mathrm{~m}$ and gray scale interval $2 \mathrm{~m}$; (b) free-air anomaly, contour interval $15 \mathrm{mGal}$ and gray scale interval $3 \mathrm{~m}$. 
os seguintes conjuntos de dados:

- Anomalia ar-livre proveniente da gravimetria marinha. Os dados foram selecionados e ajustados, de forma a minimizar as diferenças de cruzamento entre as rotas;

- Altitude da superfície oceânica (SSH) (aproximadamente igual à altura geoidal) proveniente de altimetria por satélite da missão GEOSAT (ERM), sendo selecionados, corrigidos e ajustados para minimizar as diferenças de cruzamento entre as rotas;

- Anomalia ar-livre do modelo digital de Sandwell \& Yale (1994), para preencher as regiões onde não existem nenhum dos dois tipos de dados citados acima.

Este conjunto de dados teve a componente determinística removida pelo modelo geopotencial
OSU91AF (Rapp et al., 1991) truncado em grau 180, que representa feições de longo comprimento de onda ( > $4.000 \mathrm{~km}$ ). Os erros adotados na prática para cada tipo de dado foram estimados da seguinte maneira:

- Gravimetria marinha: O valor médio das diferenças de cruzamento externo é, teoricamente, uma boa estimativa. Porém, na prática, existe a propagação de outros erros envolvidos no processamento para estimativa do campo e, desta forma, adotou-se o valor de $5 \mathrm{mGal}$ como valor representativo dos mesmos.

- Altimetria por satélite: o valor do resíduo quadrático médio dos valores das diferenças de cruzamento é igual a $0,11 \mathrm{~m}$, porém para ser coerente com a diferença entre a SSH e a altura geoidal, na prática utilizou-se o valor de $1,5 \mathrm{~m}$.

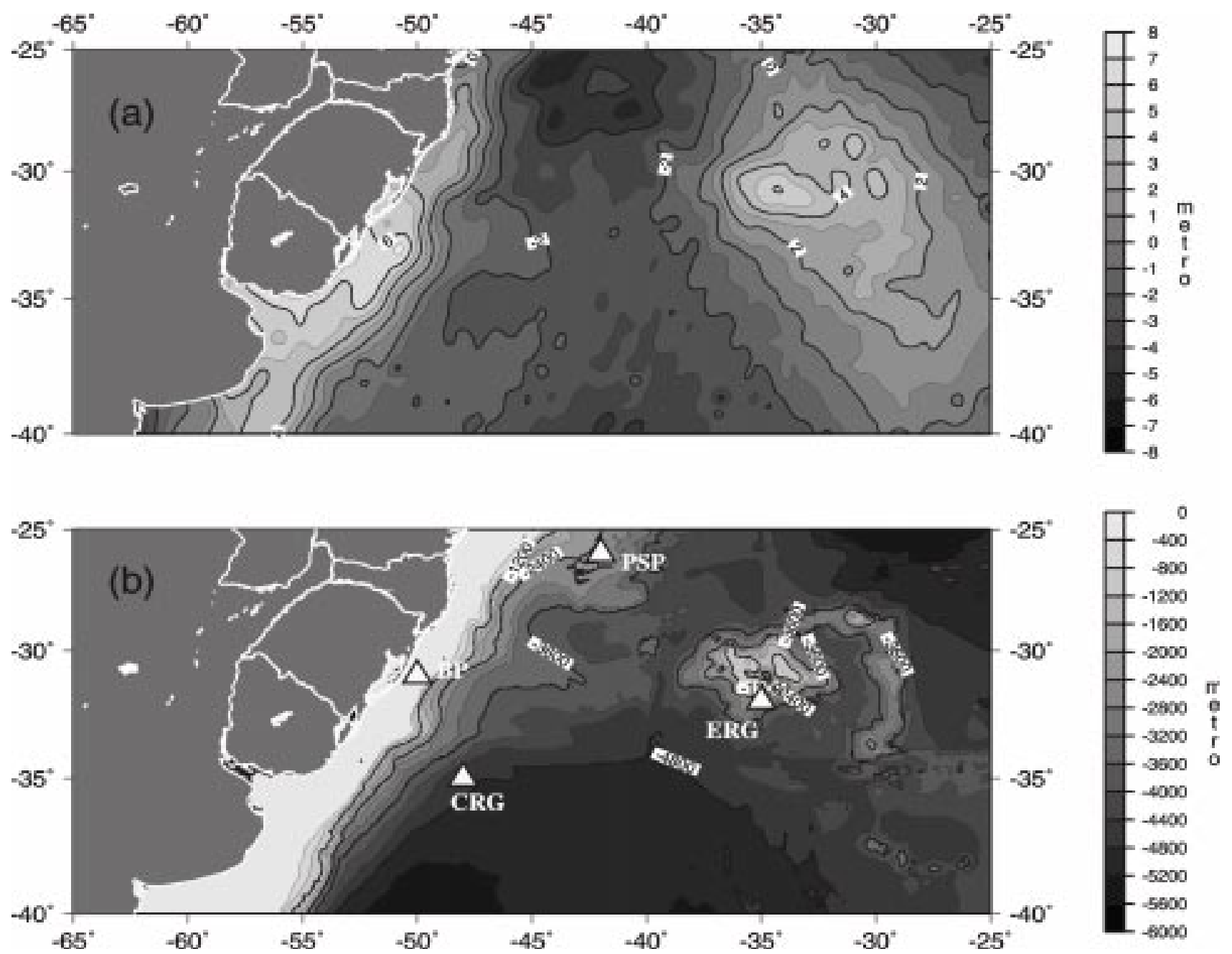

Figura 9 - (a) Anomalia do geóide, intervalo de contorno 2 m; (b) Batimetria obtida a partir do modelo digital TBASE (NGDC, 1994) onde estão indicadas as principais feições fisiográficas e tectônicas da região de estudo indicadas com triângulos: (PSP) Platô de São Paulo, (ERG) Elevação do Rio Grande, (BP) Bacia de Pelotas e (CRG) Cone do Rio Grande.

Figure 9 - (a) Residual geoid, contour interval $2 \mathrm{~m}$; (b) bathymetry from the digital model TBASE (NGDC, 1994) where the main physiographic features in the study region are indicated by triangles: (PSP) São Paulo Plateau, (ERG) Rio Grande Rise, (PB) Pelotas Basin and (CRG) Rio Grande Cone. 
- Modelo digital de Sandwell \& Yale (1994): fixouse um valor constante de $10 \mathrm{mGal}$, de forma a atribuir um peso relativamente menor a estes dados na representação integrada.

As covariâncias utilizadas na representação integrada foram obtidas pela função ajustada às covariâncias amostrais da anomalia ar-livre. Em cada compartimento de 10 ' a região circunvizinha foi dividida em 10 setores, sendo selecionados os dois pontos mais próximos do centro do compartimento em questão para cada setor. A busca dos pontos em cada setor era finalizada ao se encontrar os dois pontos mais próximos ou ao se ultrapassar o raio de busca de $1^{\circ}$. As Eqs.(10) e (11) serviram para calcular a anomalia arlivre e a altura geoidal, em blocos de 10', que são os elementos do campo de interesse neste trabalho.

A representação integrada do campo de gravidade envolveu 21.600 pontos calculados através do procedimento descrito acima e resultou na representação da anomalia ar-livre e da altura geoidal, apresentadas na Fig. 8. O mapa de batimetria com dados do modelo topográfico digital TBASE (NGDC, 1994) também foi utilizado na análise das feições observadas e este é mostrado na Fig. 9b. As incertezas nos valores calculados estão entre 10 e $15 \mathrm{mGal}$ para a anomalia ar-livre e entre 0,2 e $1 \mathrm{~m}$ para a altura geoidal, sendo menor onde a distribuição de dados é mais homogênea. No mapa de altura geoidal apresentado na Fig. 9a, foi retirado o efeito de fontes provenientes de regiões profundas no manto através de uma superfície biquadrática ajustada aos dados.

\section{Análise da representação integrada}

Para analisar a representação das anomalias ar-livre obtidas utilizando a colocação por mínimos quadrados foram feitas comparações com representações préexistentes para a região obtidos de forma independente. As representações independentes são:

a) O modelo digital de Sandwell \& Yale (1994), calculado a partir de dados de altimetria por satélite das missões GEOSAT, SEASAT e ERS-1;

b) O modelo digital de Andersen \& Knudsen (1995), obtido através de dados da missão geodésica do satélite europeu ERS-1.

A Fig.10 mostra as três representações de anomalias ar-livre para a região de estudo. Nota-se que as estruturas tectônicas principais como a Elevação do Grande, localizado na porção nordeste, as zonas de fraturas próximas a costa, a plataforma e o talude continental são todas bem definidas por essas representações.

A análise comparativa das representações do campo mostradas na Fig. 10 mostra também que os modelos digitais obtidos utilizando apenas os dados de altimetria por satélite apresentam um nível de ruído muito alto que mascaram fortemente algumas anomalias de curto e médio comprimentos de onda. Isto pode ser observado, por exemplo, na borda do Cone do Rio Grande, cuja localização (CRG) encontrase indicada na Fig. 9b. Existe um baixo gravimétrico bem destacado de direção E-W na representação integrada (Fig. 10a), e que não está bem definido na representação de Sandwell \& Yale (1994) da Fig. 10b e praticamente mascarado pelas anomalias de alta frequência observadas na representação de Andersen \& Knudsen (1995) da Fig. 10c. Na região da Elevação do Rio Grande pode-se notar uma melhor definição das estruturas de curto e médio comprimento de onda na direção NW-SE na representação integrada da Fig. $10 \mathrm{a}$.

Para melhor analisar as diferenças entre os resultados da representação integrada e aqueles baseados apenas em dados de altimetria por satélite (Sandwell \& Yale, 1994; Andersen \& Knudsen, 1995) foram construídos gráficos de anomalias ar-livre, em função da longitude, ao longo de uma rota coincidente de gravimetria marinha do cruzeiro V1809 (NGDC, 1991) situada aproximadamente ao longo da latitude $-37^{\circ}$. O perfil obtido da representação integrada (A) representa anomalias de médio e grande comprimento de onda ( $>100 \mathrm{~km}$ ) enquanto que a gravimetria marinha (B) e os dados de satélite (C) contém energia para comprimento de onda mais curtos. Nas regiões próximas à costa e na margem continental, entre -55 e $-45^{\circ}$, a representação $C$ apresenta sinal de curto comprimento de onda e com grande amplitude se comparado com os dados da representação B. Na parte central do perfil, entre -45 e $-35^{\circ} \mathrm{S}$, nota-se que as flutuações de grande amplitude na representação $C$ começam a diminuir e nota-se ai uma anomalia positiva de $25 \mathrm{mGal}$ de amplitude que não está presente nas representações A e C. A representação A filtrou os curtos comprimentos de onda e removeu esta anomalia positiva que não foi detectada no levantamento por satélite e nos levantamentos marinhos vizinhos. $\mathrm{Na}$ região de mar profundo e na região de ocorrência das zonas de fraturas, entre -35 e $-25^{\circ} \mathrm{S}$, nota-se uma boa concordância entre os dados da representação C e dados de navio enquanto que a representação integrada A tende a atenuar a amplitude das anomalias de curto comprimento que caracterizam as zonas de fratura. No mapeamento destas feições o 
recomendável é a análise dos dados ao longo das trajetórias do satélite e do navio perpendiculares ou oblíquas às fraturas. A condição de isotropia da função covariância na colocação por mínimos quadrado atenua fortemente as anomalias associadas a estas feições de tendência direcional.

\section{DISCUSSÃO}

Na discussão que se segue, serão comentadas as principais anomalias ar-livre e alturas geoidais residuais (anomalias do geóide) que resultaram da integração dos dados e estas estão mostradas nas Figs. 8(b), 9(a) e 9(b), e na sua versão colorida na capa deste volume. A estrutura crustal sob parte do Platô de São Paulo (PSP) foi modelada por Guimarães et al. (1982) utilizando as anomalias ar-livre. Este platô caracteriza-se por anomalias ar-livre negativas de amplitude até 20 mGal. O mapa de anomalias do geóide também indica uma região com anomalias de amplitude até -4 m (Fig. 9a), valores típicos de litosfera continental espessa e fria (Doin et al., 1996).

Outra feição que se destaca em todos os mapas, é a Elevação do Rio Grande (ERG). O mapa de anomalias ar-livre (Fig. 8b) mostra uma componente de longo comprimento de onda com anomalias positivas que variam de 0 a $10 \mathrm{mGal}$ e anomalias de curto comprimento de onda, cujas amplitudes podem atingir 30 mGal e que estão claramente correlacionadas com a batimetria. O mapa de anomalias do geóide (Fig. 9a) indica anomalias com até $6 \mathrm{~m}$ de amplitude. Uma análise da correlação entre elevação e altura geoidal feita por Sandwell \& MacKenzie (1989), utilizando a metodologia proposta por Haxby \& Turcotte (1978),

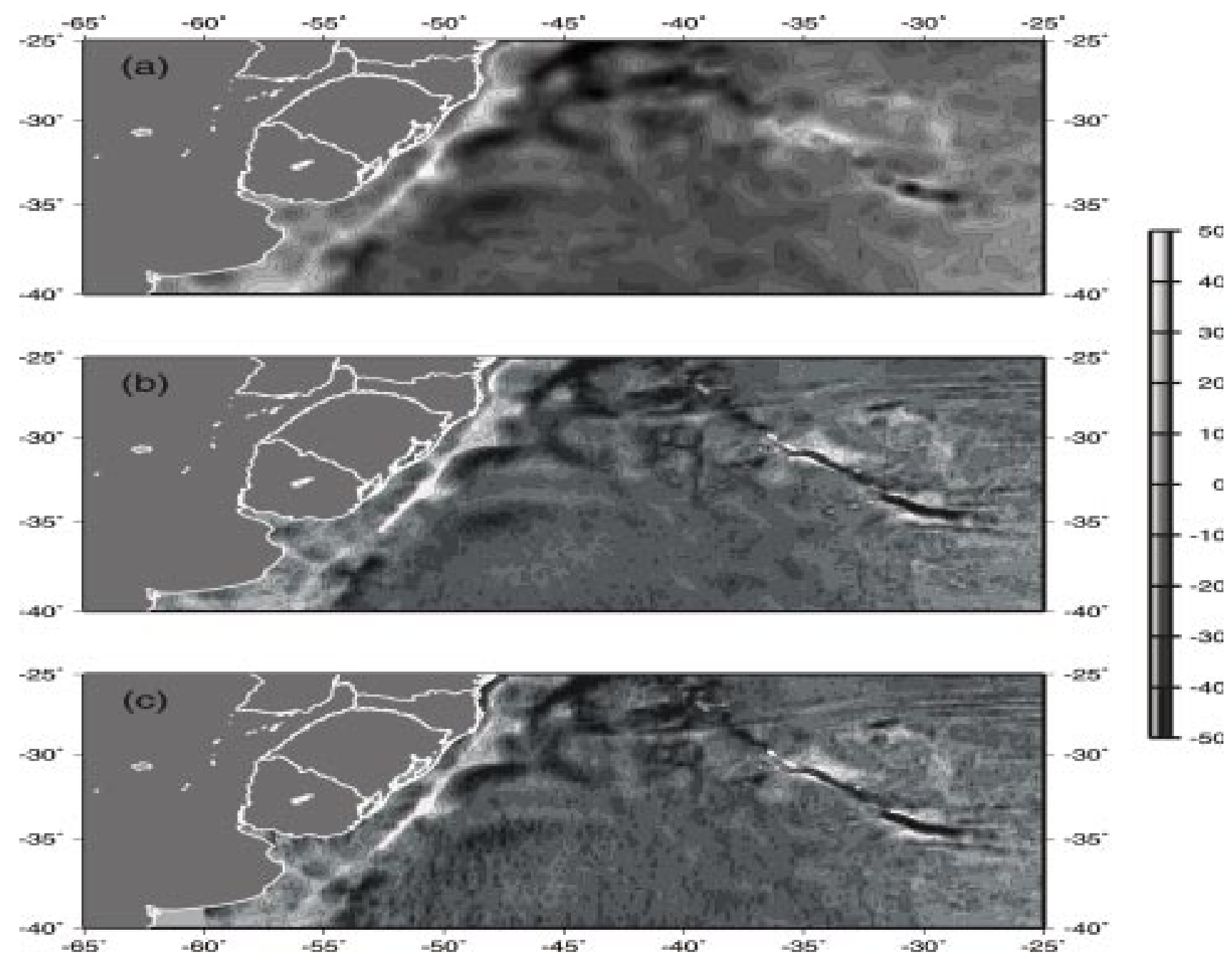

Figura 10 - Modelos digitais de anomalia ar-livre: (a) este estudo; (b) Sandwell \& Yale (1994); e (c) Andersen \& Knudsen (1995). Intervalo de contorno: $3 \mathrm{mGal}$ e intervalo da gradação em cinza: $10 \mathrm{mGal}$.

Figure 10 - Digital models of free-air anomaly: (a) this study; (b) Sandwell \& Yale (1994); and (c) Andersen \& Knudsen (1995). Contour interval: $3 \mathrm{mGal}$ and gray scale interval: $10 \mathrm{mGal}$. 

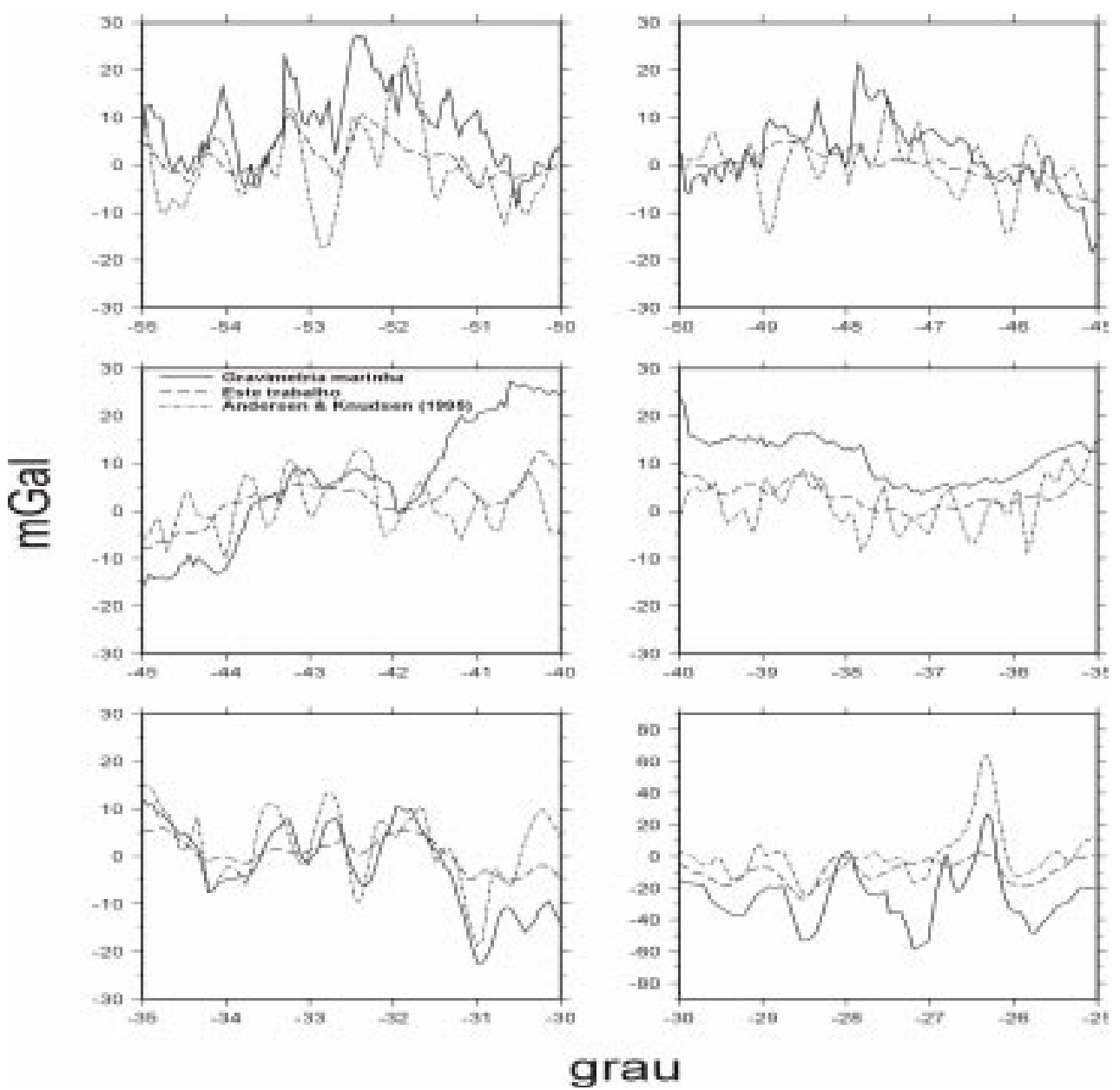

Figura 11 - Perfís de anomalias ar-livre ao longo da latitude $37^{\circ} \mathrm{S}$. Os dados ajustados de gravimetria marinha são do cruzeiro V1809 (NGDC, 1991). Note que a escala do gráfico correspondente ao segmento do perfil entre -30 e $-25^{\circ} \mathrm{W}$ foi reduzida. No texto, a representação integrada é referida como representação A.

Figure 11 - Free-air anomaly profiles along $37^{\circ} \mathrm{S}$. Adjusted shipborne gravity data are from 1809 cruise (NGDC, 1991). Note that in the graph for profile segment between -30 and $-25^{\circ} \mathrm{W}$ the vertical scale was reduced. In the text, the integrated model is referred as model $A$.

mostrou que parte desta anomalia é causada pela diminuição de densidade devido a uma anomalia térmica ainda presente sob a elevação. Segundo Gamboa \& Rabinowitz (1984), a origem da ERG está vinculada a um episódio vulcânico de grande magnitude ocorrido no Eoceno ( $40 \mathrm{Ma}$ ), portanto, a anomalia do geóide positiva indica que a perturbação térmica causada por este episódio ainda estaria presente na litosfera. Esta hipótese será futuramente testada através da modelagem quantitativa combinada das anomalias ar-livre e de geóide.

Finalmente, outra feição marcante no mapa, é a presença de uma anomalia do geóide positiva, com amplitude de 6 m (Fig. 9a e capa) que está centrada na parte leste do Escudo Rio-grandense e que se estende até o Uruguai e leste da Argentina. A porção continental desta anomalia não foi integrada no processamento deste trabalho mas resultados de Molina \& Ussami (1999) indicam que esta anomalia também ocorre na parte continental. Se anomalias do geóide positivas sugerem a existência de anomalias térmicas no manto litosférico estas devem causar uma diminuição de densidade passível de provocar um soerguimento seguido de erosão. Desta forma, a idade de resfriamento em torno de 30 Ma do escudo Rio-grandense, sugerida por datações por traço de fissão em apatita efetuadas por Hegarty et al. (1996), encontra um suporte geofísico e deve ser melhor investigada através de outras datações. A Bacia de Pelotas encontra-se totalmente situada na borda leste da anomalia do geóide, portanto, soerguimento e erosão devem estar registrados na história de subsidência desta bacia. Com efeito, a Bacia de Pelotas apresenta uma história de subsidência que se distingue das demais bacias marginais do Brasil por 
apresentar uma discordância erosional importante no Terciário (Asmus \& Ponte, 1973; Asmus \& Paim, 1986) seguido da deposição de um pacote sedimentar expressivo desde o Mioceno. A influência do aparecimento de uma anomalia térmica em torno de 60 e 30 Ma e sua posterior evolução pode ser modelada e deve ser levada em conta quando do estudo da subsidência deste segmento da margem continental. Resultados de retrodenudação (backstripping) em alguns poços da Bacia de Pelotas efetuado por Fontana (1990) indicam que o modelo termo-mecânico de McKenzie (1978) não explica a história de subsidência da bacia após o Cretáceo Superior.

\section{CONCLUSÃO}

Os dados de altimetria por satélite, provenientes da missão GEOSAT (ERM), apresentam distância relativamente grande entre as rotas. Assim, o uso isolado dos mesmos não permite calcular modelos gravimétricos de alta resolução. Os dados de gravimetria marinha ajudam a adensar a distribuição total dos dados e permitem atenuar este problema em determinadas áreas, notadamente em regiões próximas à costa e com lâmina d'água menor do que $1.000 \mathrm{~m}$, onde os dados de altimetria por satélite não são confiáveis. Além disso, os resultados ora obtidos mostram que os modelos gravimétricos baseados apenas em dados de altimetria por satélite apresentam problemas de ruído que podem mascarar feições gravimétricas de curto comprimento de onda.

A representação integrada calculada neste trabalho define melhor algumas feições tectônicas tais como a porção sul do Platô de São Paulo e o Cone do Rio Grande e apresenta resolução efetiva maior nas regiões próximas à costa. Apesar disso, esta metodologia é problemática para o mapeamento de estruturas lineares de curtos comprimentos de onda, como por exemplo, as zonas de fraturas. Novos dados de altimetria por satélite, como os referentes às missões TOPEX-POSEIDON e ERS-1, que possuem espaçamento menor entre as rotas, podem ser incorporados no processamento com o intuito de melhorar a resolução efetiva. Integrando estes novos dados aos dados de gravimetria marinha e gravimetria continental, pode-se aplicar a metodologia de colocação por mínimos quadrados a fim de obter um aprimoramento da resolução e precisão dos modelos gravimétricos e o modelamento das principais estruturas tectônicas da região.

\section{AGRADECIMENTOS}

Agradecemos à FAPESP pelos recursos concedidos através dos processos 90/3061-7 e 97/00948-0 (bolsa de Iniciação Científica de Emilson Pereira Leite), e ao PADCT/FINEP (proc. 65.92.0070.00). Desejamos agradecer também aos dois revisores anônimos pelas valiosas sugestões e pelos comentários.

\section{REFERÊNCIAS}

ANDERSEN, O. B. \& KNUDSEN, P. -1995-Global altimetric gravity map from the ERS-1 geodetic mission (cycle 1). Earth Observation Quarterly, 47: 1-5.

ASMUS, H. E. \& PONTE, F. C. -1973- The Brazilian marginal basins. In The Ocean basins and margins, The South Atlantic, Vol. 1 (A.E.M. Nairn \& F.G. Stehli, eds.). Plenum Press, NY, 87-133.

ASMUS, H. E. \& PAIM, P. S. G. -1986- Condicionamento geológico na evolução da planície costeira sulRiograndense. Anais do XXXIV Congresso Brasileiro de Geologia. Sociedade Brasileira de Geologia, 1: 461471, Goiânia, GO.

CHENEY, R.E,DOUGLAS, B.C.,AGREEN, R.W.,MILLER L., PORTER D. L. \& DOYLE, N. S. -1987- Geosat altimeter geophysical data record user handbook. Tech. Memo. NOS NGS-46. Natl. Oceanic and Atmos. Admin., Boulder, Colo, 30pp.

DEHLINGER, P. -1978- Marine Gravity. Elsevier, N.Y., 322pp.

DOIN, M.P., FLEITOUT,L. \& MCKENZIE, D. -1996-Geoid anomalies and the structure of continental and oceanic lithospheres. J. Geophys. Res., 101: 16.119-16.135.

FONTANA, R. L. -1990- Desenvolvimento termo-mecânico da Bacia de Pelotas e parte sul da plataforma de Florianópolis. In Origem e Evolução de Bacias Sedimentares (G.P. Raja-Gabaglia \& E.J. Milani, eds.), PETROBRÁS, Rio de Janeiro, 377-400.

GAMBOA, L. A. P. \& RABINOWITZ, P. D. -1984- The evolution of the Rio Grande Rise in the Southwest Atlantic Ocean. Marine Geology, 58: 35-58.

GUIMARÃES, P.P.D, ALMEIDA, H.P., KOWSMANN, R. O. \& COSTA, M. P. A. -1982- Modelagem gravimétrica na porção sul do platô de São Paulo e suas implicações geológicas. Anais do XXXII Congresso Brasileiro de Geologia, SBG, 4: 1570-1575. Salvador, BA.

HAXBY, W. F. \& TURCOTTE, D. L. -1978- On isostatic geoid anomalies. J. Geophys. Res., 83:5473-5478.

HEGARTY, K. A., DUDDY, I. R. \& GREEN,P. F. -1996- The thermal history in around the Paraná Basin using Apatite Fission Track Analysis - Implications for Hydrocarbon Occurrences and Basin formation. In Alkaline Magmatism in Central-Eastern Paraguay: Relationships with Coeval Magmatism in Brazil. (P. Comin-Chiaramonti \& C.B. Gomes, eds), EDUSP/FAPESP, São Paulo, 67-84.

HEISKANEN, W. A. \& MORITZ, H. -1967- Physical Geodesy. Freeman, San Francisco, 364 pp.

HITTLEMAN, A. M., GROMAN, R. C., HAWORTH, R. T., 
HOLCOMBE, T.L., MCHENDRIE, G. \& SMITH,S.M. -1977- The Marine geophysical Data Exchange Format MGD77, Key to Geophysical Records Documentation 10, National. Geophysical Data Center, Natl. Ocean. and Atmos. Admin., Boulder, Colo.

KNUDSEN, P. -1987- Estimation and modeling of the local empirical covariance function using gravity and satellite altimetry data. Bull. Géod., 61:145-160.

McKENZIE, D. P. -1978- Some remarks on the development of sedimentary basins. Earth Plan. Sci. Lett., 40:25-32.

MOLINA, E. C. -1996- Ajustamento e integração de dados gravimétricos e de altimetria por satélite na representação do campo de gravidade no Atlântico Sul. Tese de doutoramento, IAG-USP, $198 \mathrm{pp}$.

MOLINA, E. C. \& SÁ, N. C. DE -1993- Avaliação da precisão e resolução dos dados do SEASAT em relação aos do projeto EQUANT. III Congr. Intern. da Soc. Bras. de Geofísica, Resumos Expandidos, 2:1225-1230. Rio de Janeiro, RJ

MOLINA, E. C. \& USSAMI, N. -1999- The Geoid in SE Brazil and adjacent regions: new constraints on density distribution and thermal state of the lithosphere. J. Geodyn., 28:357-374.

MORITZ, H. -1980- Advanced Physical Geodesy. Abacus Press, Wichmann, 500 pp.

NEUMANN, G. A., FORSYTH, D. W. \& SANDWELL, D. 1993- Comparison of marine gravity from shipboard and high-density satellite altimetry along the midatlantic ridge, $30.5^{\circ}-35.5^{\circ} \mathrm{S}$. Geophys. Res. Lett., 20(15): 1639-1642.

NGDC (NATIONAL GEOPHYSICAL DATA CENTER) 1991- GEODAS Marine Geophysical Data, Bathymetry, Magnetics, Gravity, Volume 1, Data (CD-ROM), U.S. Department of Commerce, NOAA, Colorado, USA.

NGDC (NATIONAL GEOPHYSICAL DATA CENTER) 1994- Terrain Base, World Digital Terrain Data (CDROM), Release 1.0, U.S. Department of Commerce, NOAA, Colorado, USA.

PRINCE, R. \& FORSYTH, D. W. -1984- A simple objective method for minimizing crossover errors in marine gravity data. J. Geophys. Res., 49: 1070-1083.

RAPP, R. H. \& PAVLIS, N. K. -1990- The development of an isostatic gravitational model to degree 360 and its use in global gravity modeling. Geophys. J. Int., 100: 369-378.

RAPP, R. H., WANG, Y. M. \& PAVLIS, N. K. -1991- The Ohio State 1991 geopotential and sea surface topography harmonic coefficient models. Report 410, Dept. Geod. Sci. Surveying, Ohio State Univ., Columbus.

SÁ, N. C. DE -1988- Modelos estatísticos e representação integrada do campo de gravidade no Brasil. Tese de doutoramento, IAG-USP, 219 pp.

SÁ, N. C. DE, USSAMI, N. \& MOLINA, E. C. -1993-Gravity map of Brazil 1. Representation of free-air and Bouguer anomalies. J. Geophys. Res., 98: 2187-2197.

SANDWELL, D. T. \& MACKENZIE, K. R. -1989- Geoid height versus topography for oceanic plateaus and swells. J. Geophys. Res., 94: 7403-7418.

SANDWELL, D.T. \& McADOO, D. C. -1990- High-accuracy, high-resolution gravity profiles from 2 years of the Geosat Exact Repeat Mission. J. Geophys. Res., 95: 3049-3060.

SANDWELL, D. T. \& YALE, M. M. -1994-ERS-1 Geodetic Mission Reveals Detailed Tectonic Structures. AGU Fall Meeting, San Francisco, Abstracts, pp. 155.

TSCHERNING, C. C. \& RAPP, R. H. -1974- Closed covariances expressions for gravity anomalies, geoid undulation and deflections of the vertical implied by the anomaly degree variance models. Report $\mathrm{n}^{\circ} 208$, The Ohio State Universtiy, Columbus, 89 pp.

WESSEL, P. \& WATTS, A. B. -1988- On the accuracy of marine gravity measurements. J. Geophys. Res., 93: 393413. 


\section{INTEGRATION OF SHIPBORNE GRAVITY AND SATELLITE ALTIMETRY (GEOSAT/ERM) IN THE SOUTH ATLANTIC $\left(65 / 25^{\circ} \mathrm{W}\right.$ AND $\left.25 / 40^{\circ} \mathrm{S}\right)$}

Conventional shipborne gravity data were integrated with gravity data derived from satellite altimetry over a region in the South Atlantic Ocean situated between $65 / 25^{\circ} \mathrm{W}$ and $25 / 40^{\circ} \mathrm{S}$. This region includes physiographic and tectonic features such as the São Paulo Plateau, Rio Grande Rise, Pelotas and Argentina Basins. Least squares collocation was used in order to obtain free-air anomaly and geoid height models, with nominal resolution of 10'. Shipborne gravity data was mostly obtained from GEODAS data bank updated until 1995 and the satellite derived gravity data are from one year coverage of GEOSAT/Exact Repeat Mission. Initially, the shipborne data were corrected and adjusted for cross-over errors which reduced the initial 120 to 93 cruises. The final data bank comprises 36,854 adjusted marine gravity stations. Satellite altimetry derived gravity data were selected after preprocessing to eliminate data over land and ice and with large error after instrumental correction. Cross-over adjustment was also applied to the data. The final selection of data was carried out by comparing each sea surface height (SSH) with geoid height predicted by a global geopotencial model. Data points with absolute value differences higher than $20 \mathrm{~m}$ were discarded. Also, data points with SSH absolute values higher than $100 \mathrm{~m}$ were discarded. Altogether, satellite derived data sums 10,435 points with average spacing of $7 \mathrm{~km}$ along track, approximately $150 \mathrm{~km}$ between tracks and r.m.s. value of $0.11 \mathrm{~m}$ after adjustment. Least squares collocation was subsequently applied in order to combine shipborne and satellite gravity data. First, the deterministic component was removed using the OSU91AF geopotencial model, truncated at degree 180. Weight for each data source was assigned using an estimated error of $5 \mathrm{mGal}$ for marine gravity, $1.5 \mathrm{~m}$ for sea surface height and finally, $10 \mathrm{mGal}$ for Sandwell \& Yale's (1994) digital model in order to give a lower weight in the integrated model. The free-air anomaly model obtained in this work was compared with two other recent models derived only from satellite altimetry (Sandwell \& Yale, 1994; Andersen \& Knudsen, 1995). The results show that the methodology used in the present study produces gravity models for the oceans free of noise. These high frequency components are observed in almost all models based solely on satellite altimetry. Apart from this result, the integration of conventional marine gravity data is fundamental to improve the gravity field in water depth shallower than $1,000 \mathrm{~m}$, such as near the coast and continental margins, where satellite data are not reliable.

The free-air anomaly map obtained in this study revealed previously unknown gravity anomalies such as an E-W trending gravity low south of Rio Grande Fan, between $50 / 45^{\circ} \mathrm{W} 34 / 37^{\circ} \mathrm{S}$. Also, the residual geoid map revealed two geoid highs, the first over the Rio Grande Rise and the second over the Rio Grandense Shield and its adjoinning continental margin, including the Pelotas Basin. The two positive geoid anomalies indicate areas of recent ( 60 - $40 \mathrm{Ma}$ ) thermal reactivation. The oceanic lithosphere between the continental margin and the Rio Grande Rise is characterized by negative free-air and geoid anomalies as predicted by the thermal model for an older and thicker lithosphere. Further quantitative interpretation of these anomalies are under way.

\section{NOTA SOBRE OS AUTORES}

Emilson Pereira Leite

Bacharel em Geofísica em 1997 pelo IAG/USP atualmente no programa de Mestrado em Geofísica no IAG/USP. Áreas de interesse: Altimetria por satélite, Métodos Potenciais e Tectonofísica.
Naomi Ussami

Docente no IAG/USP desde 1981, PhD em Geofísica na Universidade de Durham, Inglaterra em 1986; Pós-doutorado na Universidade de Cornell em 1995. Áreas de interesse métodos potenciais aplicados a estudos tectônicos e litosféricos.

\section{Eder Cassola Molina}

Bacharel em Geofísica em 1987; Mestrado em Geofísica em 1990 e Doutorado em Geofísica em 1996 e docente no Depto. de Geofísica no IAG/USP desde 1988. Áreas de interesse: Altimetria por Satélite, Métodos Potenciais e Processamento de dados aerogeofísicos. 